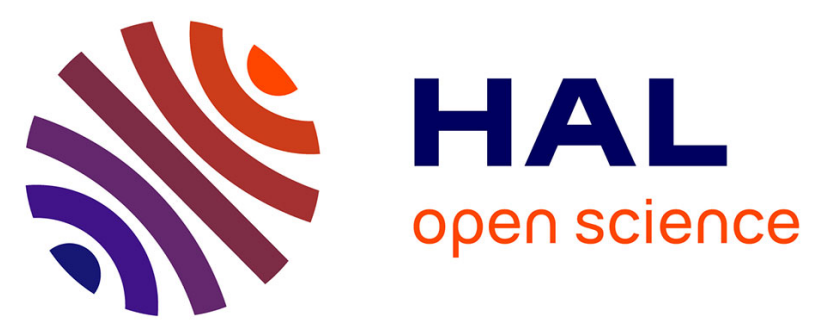

\title{
Quelles stratégies pour un maintien de la polyculture-élevage? Une étude des trajectoires passées d'exploitations dans les coteaux de Gascogne
}

Julie Ryschawy, Norma Choisis, Jean Philippe Choisis, Alexandre Joannon, Annick Gibon

\section{To cite this version:}

Julie Ryschawy, Norma Choisis, Jean Philippe Choisis, Alexandre Joannon, Annick Gibon. Quelles stratégies pour un maintien de la polyculture-élevage? Une étude des trajectoires passées d'exploitations dans les coteaux de Gascogne. L'agriculture en famille: travailler, réinventer, transmettre, EDP Sciences, 382 p., 2014, 978-2-7598-1192-2. hal-01210114

\section{HAL Id: hal-01210114 \\ https://hal.science/hal-01210114}

Submitted on 5 Jun 2020

HAL is a multi-disciplinary open access archive for the deposit and dissemination of scientific research documents, whether they are published or not. The documents may come from teaching and research institutions in France or abroad, or from public or private research centers.
L'archive ouverte pluridisciplinaire HAL, est destinée au dépôt et à la diffusion de documents scientifiques de niveau recherche, publiés ou non, émanant des établissements d'enseignement et de recherche français ou étrangers, des laboratoires publics ou privés.

\section{(1) (1) $\$$}

Distributed under a Creative Commons Attribution - NonCommercial - NoDerivatives 44.0 


\title{
Quelles stratégies pour un maintien de la polyculture-élevage ? Une étude des trajectoires passées d'exploitations dans les coteaux de Gascogne
}

\author{
Julie Ryschawy ${ }^{1}$, Norma Choisis ${ }^{2}$, Jean-Philippe Choisis ${ }^{3}$, \\ Alexandre Joannon ${ }^{4}$, Annick Gibon ${ }^{5}$
}

\section{Introduction}

La polyculture-élevage est de plus en plus reconnue au plan international comme un moyen de limiter les problèmes environnementaux tout en permettant une agriculture productive et économiquement viable (Russelle et al., 2007 ; Wilkins, 2008). Ces systèmes intégrant des cultures et de l'élevage à l'échelle de l'exploitation ont été pourtant largement marginalisés dans les pays développés. La mondialisation des échanges adossée à une libéralisation des marchés a fortement incité à des économies d'échelle et à la spécialisation des exploitations et des territoires (Mazoyer et Roudart, 1997).

En Europe, en particulier, les aides de la PAC et la diminution de la main-d'œuvre agricole ont favorisé la spécialisation et marginalisé la polyculture-élevage (EC, 1999 ; Meynard, 2012). La spécialisation des exploitations et des régions est telle aujourd'hui qu'elle produit de nombreuses externalités négatives, e.g. pollution des nappes phréatiques, érosion de la biodiversité, simplification des paysages, coût élevé du transport des intrants et des produits (Wilkins, 2008 ; Chatellier et Gaigné, 2012). Un enjeu fort est donc de comprendre les conditions conduisant au maintien ou au non-maintien de la polyculture-élevage en Europe. Pour comprendre ces conditions de maintien, il est fondamental d'appréhender leur évolution sur le temps long. Selon Dedieu (2009), illustrer la diversité des " chemins » de l'action sur le long terme en situation d'incertitude permet de voir les tensions qui influent sur la résilience des systèmes, i.e. leur capacité à résister aux aléas. Nous faisons dès lors l'hypothèse que les agriculteurs qui se sont maintenus en polyculture-élevage

1. INRA, UMR 1248 AGIR, 31324 Castanet-Tolosan, France, julie.ryschawy@ensat.fr

2. INRA, UMR 1201 Dynafor, 31324 Castanet-Tolosan, France

3. INRA, UMR 1201 Dynafor, 31324 Castanet-Tolosan, France, jean-philippe.choisis@toulouse.inra.fr

4. INRA, UR 0980 SAD Paysage, 35000 Rennes, France, joannon@rennes.inra.fr

5. INRA, UMR 1201 Dynafor, 31324 Castanet-Tolosan, France, gibon@toulouse.inra.fr

Ce chapitre de l'ouvrage L'agriculture en famille : travailler, réinventer, transmettre est publié en Open Access sous licence creative commons CC-BY-NC-ND permettant l'utilisation non commerciale, la distribution, la reproduction 
ont développé des capacités adaptatives spécifiques en situation d'incertitude. Cette étude vise à identifier et interpréter les stratégies adaptatives des agriculteurs leur permettant de maintenir des exploitations de polyculture-élevage sur le long terme.

\section{Analyser les « chemins pour durer " des agriculteurs}

\section{Une zone d'étude dans laquelle la polyculture-élevage s'est maintenue}

Notre démarche générale a été appliquée à une étude de cas régionale : les coteaux de Gascogne, au sud-ouest de Toulouse en région Midi-Pyrénées. Il s'agit d'une situation de zone défavorisée simple, européenne, où les politiques de modernisation de l'agriculture n'ont pas entraîné une forte spécialisation des exploitations. Les exploitations de polyculture-élevage, ancrées localement dans une société rurale organisée en "système à maisons ", avec un seul héritier par famille et une logique d'autosubsistance familiale, y ont persisté (Sourdril et al., 2006). Ce fonctionnement a permis de conserver des paysages bocagers en mosaïque combinant cultures, prairies et éléments boisés. Le zonage agro-écologique mondial (FAO, 1995) classifie la région comme une zone tempérée avec des sécheresses estivales fréquentes. Ces conditions climatiques alliées à des sols de type argilo-calcaires et de fortes pentes ont induit une dominance de prairies dans la $S A U^{6}$ régionale.

Le site d'étude retenu est composé de quatre villages, ce qui représente 61 exploitations sur environ 4000 hectares de SAU. Il s'agit d'un site de recherche socioécologique à long terme de l'UMR Dynafor qui fait partie du réseau européen LTER (European Long-Term Ecological Research). Les relations de long terme entre paysage, agriculture et biodiversité y sont étudiées en partenariat avec les acteurs locaux. Les résultats présentés ici proviennent de l'étude de la dynamique locale de l'agriculture effectuée au sein d'un groupe local de recherche en partenariat associant l'UMR Dynafor, les municipalités des quatre communes, l'ACVA (Association cantonale de vulgarisation agricole) d'Aurignac, les agriculteurs du site d'étude et la Chambre départementale d'agriculture de Haute-Garonne. La collaboration au sein de ce groupe a porté sur toutes les étapes des différents volets des travaux, du choix de leurs orientations et de leurs méthodologies à l'élaboration et à la discussion critique des résultats (Choisis et al., 2010 ; Ryschawy, 2012).

Parmi les exploitations du site d'étude, trois types de systèmes de production spécialisés coexistent avec les systèmes de polyculture-élevage : les systèmes bovins laitiers, bovins allaitants et grandes cultures. Les exploitations spécialisées en grandes cultures sont principalement situées en fonds de vallée ; les autres exploitations ont des terres qui sont soit situées sur les coteaux soit réparties entre fonds de vallée et coteaux. Un maintien d'exploitations de polyculture-élevage alliant bovins allaitants

6. SAU : surface agricole utile. 
et grandes cultures y est observé ( $47 \%$ des exploitations actuelles, Choisis et al., 2010). Ce type de polyculture-élevage sera le support de notre étude. Les caractéristiques principales des exploitations du cas d'étude sont fournies dans le tableau 1.

Tab. 1. Caractéristiques principales des exploitations du site d'étude selon leurs types de systèmes de production (Ryschawy et al., 2012).

\begin{tabular}{lcccc}
\hline & Bovin laitier & $\begin{array}{c}\text { Bovin } \\
\text { allaitant }\end{array}$ & $\begin{array}{c}\text { Grandes } \\
\text { cultures }\end{array}$ & $\begin{array}{c}\text { Polyculture- } \\
\text { élevage }\end{array}$ \\
\hline Nombre d'exploitations & $6(12 \%)$ & $12(25 \%)$ & $7(15 \%)$ & $23(48 \%)$ \\
SAU (ha) & & & & \\
- Moyenne \pm écart-type & $93 \pm 52$ & $89 \pm 58$ & $66 \pm 58$ & $118 \pm 82$ \\
- Minimum/Maximum & {$[29 ; 157]$} & {$[28 ; 196]$} & {$[15 ; 139]$} & {$[37 ; 218]$} \\
SFP (ha) & & & & \\
- Moyenne \pm écart-type & $48 \pm 32$ & $77 \pm 53$ & $13 \pm 12$ & $64 \pm 56$ \\
- Minimum/Maximum & {$[19 ; 97]$} & {$[28 ; 180]$} & {$[2 ; 34]$} & {$[12 ; 127]$} \\
$\begin{array}{l}\text { UTA } \\
\text { - Moyenne } \pm \text { écart-type }\end{array}$ & $2,3 \pm 1,1$ & $1,3 \pm 0,6$ & $0,5 \pm 0,4$ & $1,8 \pm 1,0$ \\
- Minimum/Maximum & {$[1 ; 4]$} & {$[1 ; 2]$} & {$[0,5 ; 1]$} & {$[1 ; 4]$} \\
Nombre de vaches & & & & \\
- Moyenne \pm écart-type & $48 \pm 20$ & $54 \pm 36$ & n.a. & $48 \pm 37$ \\
- Minimum/Maximum & {$[20 ; 70]$} & {$[20 ; 120]$} & n.a. & {$[20 ; 135]$} \\
\hline
\end{tabular}

SAU : surface agricole utile, SFP : surface fourragère principale, UTA : unité de travail agricole Les exploitations de polyculture-élevage locales allient bovins allaitants et grandes cultures. Elles sont définies selon la définition de Seré et al. (1996).

Pour chaque caractéristique, les moyennes \pm écarts-types sont donnés. Pour une description plus précise, les minimum et maximum sont donnés pour chaque indicateur considéré.

\section{Une enquête historique de l'évolution des pratiques des agriculteurs}

Une démarche d'enquêtes exhaustive a été appliquée au site d'étude fin 2006-2007: la totalité des ménages ayant une activité agricole et utilisant des terres sur le territoire est considérée. La méthode d'enquête pour l'étude intégrée et "spatialement explicite " du fonctionnement et des changements sociotechniques des exploitations a été adaptée de Mottet et al. (2006). La spatialisation de la gestion agricole de l'espace et de ses modifications de la parcelle au paysage est aujourd'hui vue comme essentielle dans l'étude des changements des agricultures locales en raison de l'importance des questions environnementales (Gibon et al., 2010). 
Les données collectées ont porté sur la structure des exploitations, l'usage des sols, les pratiques des agriculteurs ainsi que leur évolution de 1950 à 2005. Les données ont été stockées dans une base de données spatialisée, DynafarmCoto®. 56 agriculteurs ont pu être enquêtés parmi les 61 ayant des terres sur le site d'étude (Choisis et al., 2010).

Des données socioéconomiques ont, par ailleurs, été collectées au niveau municipal, cantonal et régional sur les indicateurs de marchés, les politiques agricoles, les dynamiques des exploitations et des filières locales (statistiques nationales officielles, Agreste, 2010) et des entretiens d'acteurs locaux dont des conseillers agricoles, des maires et responsables de coopératives ont été conduits (Belland, 2011).

\section{Une typologie de trajectoires passées des exploitations}

L'étude se fonde sur l'analyse des trajectoires passées des exploitations afin d'en caractériser la résilience (Milestad et Darnhofer, 2003). Nous mobilisons les approches typologiques des trajectoires d'exploitation initialement proposées par Capillon et Manichon (1979) selon lesquelles la structure, le fonctionnement et l'évolution des exploitations doivent être prises en compte dans leur dimension historique afin d'appréhender leur avenir. Pour aborder les changements survenus dans les exploitations, nous nous basons sur les cadres de représentation des changements passés des systèmes d'élevage proposés par Mottet et al. (2006) et Moulin et al. (2008). Nous avons ainsi développé une méthodologie pour extraire des types de trajectoires à partir de données historiques collectées en enquêtes, comme l'indiquent Dobremez et Bousset (1996) et Rueff et al. (2012). Cette méthode permet de simplifier la réalité pour l'interpréter.

La méthode d'étude des trajectoires passées des exploitations que nous avons mise en place se décompose en deux étapes. Nous avons, tout d'abord, réalisé une analyse visuelle des trajectoires des 50 exploitations pour lesquelles les données historiques collectées étaient suffisantes. Pour cela, nous avons construit une méthode d'analyse graphique des trajectoires individuelles d'exploitations (Fig. 1). L’analyse a été réalisée sur la période 1950-2005. Nous avons décrit l'évolution des caractéristiques des exploitations à des dates sélectionnées selon un pas de temps de dix ans, qui nous permettait de capter les évolutions principales sur la période. 


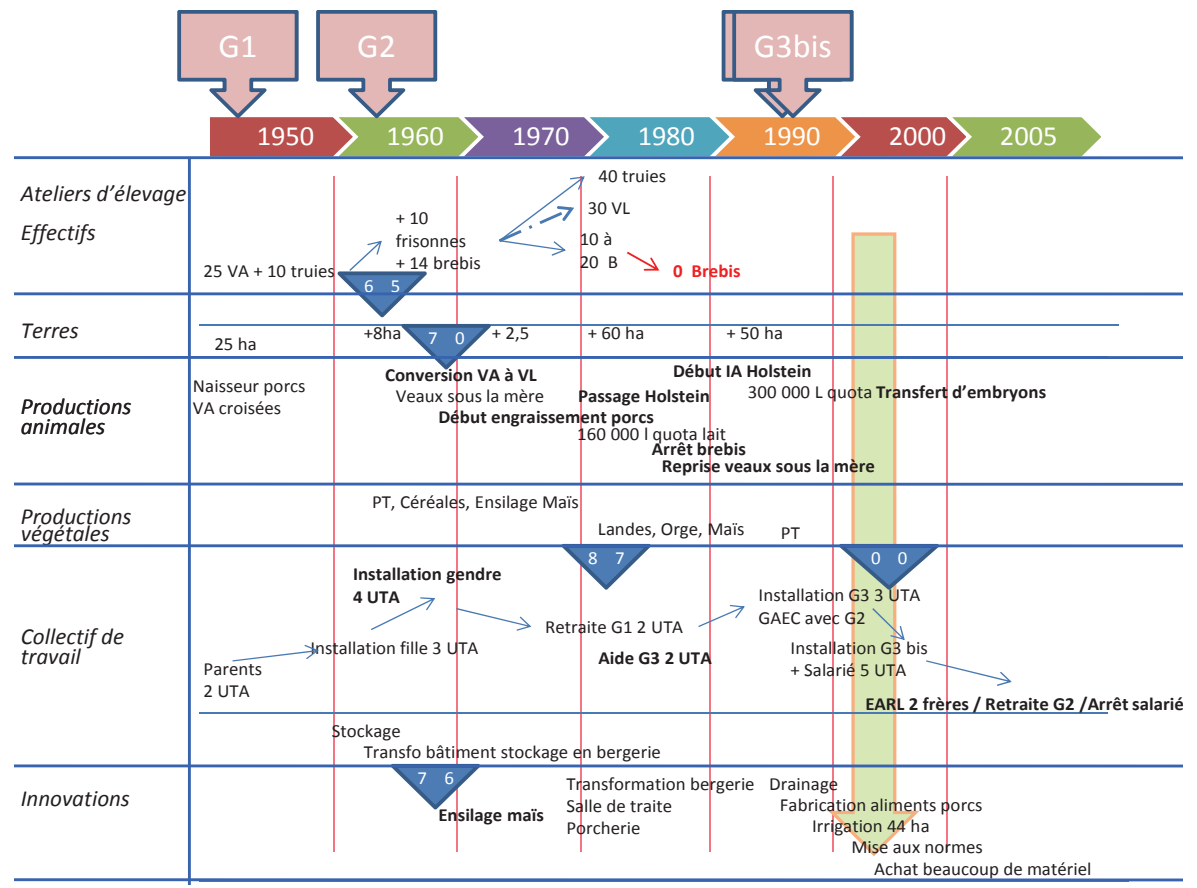

Les évènements sont présentés par décennie de 1950 à 2005 et classés selon six catégories principales. Des flèches permettent d'indiquer l'évolution des variables quantitatives. Des triangles roses indiquent l'arrivée des différentes générations d'agriculteurs. Des triangles bleus marquent les évènements les plus marquants selon l'agriculteur et précisent l'année correspondante. Une flèche verte indique un évènement ayant induit une modification marquante du fonctionnement du système.

Fig. 1. Exemple anonymisé de représentation graphique individuelle d'une trajectoire d'exploitation (adapté de Ryschawy, 2012).

À partir de l'examen de l'ensemble de la base de données, nous avons sélectionné un premier jeu de 30 indicateurs de structure et de fonctionnement que nous avons classés en six catégories principales : les terres, les productions animales et effectifs, les productions végétales, le collectif de travail et les investissements dans les bâtiments et aménagements. Parmi les 30 indicateurs retenus, certains n'étaient toutefois pas discriminants comme la présence de prairies permanentes, que l'on trouve dans toutes les exploitations. La comparaison des graphiques individuels des 50 trajectoires nous a permis de sélectionner in fine 20 indicateurs pouvant rendre compte des différences d'évolution entre les exploitations (Tab. 2). Sur la base de ces 20 indicateurs, nous avons effectué, dans une seconde étape, une typologie de trajectoires à l'aide d'analyses multivariées selon une méthode inspirée de Gibon et al. (1999a), Garcia-Martinez et al. (2009) et Rueff et al. (2012) et fondée sur l'analyse statistique combinée de phénomènes temporels et stationnels développée en écologie par Doledec et Chessel (1987). 
Tab. 2. Variables retenues pour l'analyse multivariée des trajectoires d'exploitation.

\begin{tabular}{|c|c|c|c|}
\hline Catégorie & Indicateur de & Variable utilisée & Type \\
\hline \multirow[t]{2}{*}{ Terres } & Taille totale de l'exploitation & Surface Agricole Utile (SAU) & Quantitative \\
\hline & Type de mode de faire-valoir & $\begin{array}{l}\text { Pourcentage de la SAU en } \\
\text { fermage }\end{array}$ & Quantitative \\
\hline \multirow[t]{3}{*}{$\begin{array}{l}\text { Collectif } \\
\text { de travail }\end{array}$} & Taille du collectif de travail & $\begin{array}{l}\text { Nombre d'Unités de Travail } \\
\text { Agricole (UTA) }\end{array}$ & Quantitative \\
\hline & $\begin{array}{l}\text { Changement de génération } \\
\text { d'agriculteurs }\end{array}$ & $\begin{array}{l}\text { Arrivée d'une nouvelle } \\
\text { génération }\end{array}$ & Qualitative \\
\hline & $\begin{array}{l}\text { Nombre de générations } \\
\text { travaillant conjointement }\end{array}$ & $\begin{array}{l}\text { Nombre de générations en } \\
\text { activité }\end{array}$ & Quantitative \\
\hline \multirow[t]{4}{*}{ Ateliers } & $\begin{array}{l}\text { Atelier de production de } \\
\text { bovins allaitants }\end{array}$ & Présence de bovins allaitants & Qualitative \\
\hline & Atelier de production laitière & Présence de bovins laitiers & Qualitative \\
\hline & Cultures de vente & Présence de cultures de vente & Qualitative \\
\hline & $\begin{array}{l}\text { Diversification de } \\
\text { l'exploitation }\end{array}$ & Nombre d'ateliers & Quantitative \\
\hline \multirow[t]{3}{*}{$\begin{array}{l}\text { Productions } \\
\text { animales }\end{array}$} & Taille du troupeau bovin & $\begin{array}{l}\text { Nombre de mères dans le } \\
\text { troupeau bovin }\end{array}$ & Quantitative \\
\hline & $\begin{array}{l}\text { Innovation pour } \\
\text { l'alimentation animale }\end{array}$ & Utilisation d'ensilage de maïs & Qualitative \\
\hline & Orientation de l'atelier bovin & $\begin{array}{l}\text { Race principale du troupeau } \\
\text { bovin }\end{array}$ & Qualitative \\
\hline \multirow[t]{4}{*}{$\begin{array}{l}\text { Productions } \\
\text { végétales }\end{array}$} & $\begin{array}{l}\text { Intensification des pratiques } \\
\text { culturales }\end{array}$ & $\begin{array}{l}\text { Présence de maïs pour la } \\
\text { vente }\end{array}$ & Qualitative \\
\hline & $\begin{array}{l}\text { Innovation dans la gestion des } \\
\text { prairies }\end{array}$ & Présence d'ensilage d'herbe & Qualitative \\
\hline & $\begin{array}{l}\text { Utilisation de prairies } \\
\text { temporaires }\end{array}$ & $\begin{array}{l}\text { Présence de prairies } \\
\text { temporaires }\end{array}$ & Qualitative \\
\hline & $\begin{array}{l}\text { Adoption de cultures } \\
\text { innovantes }\end{array}$ & Adoption de soja ou tournesol & Qualitative \\
\hline \multirow[t]{4}{*}{ Investissements } & Aménagement des terres & Réalisation du drainage & Qualitative \\
\hline & $\begin{array}{l}\text { Investissements pour les } \\
\text { cultures }\end{array}$ & Utilisation de l'irrigation & Qualitative \\
\hline & $\begin{array}{l}\text { Investissements pour les } \\
\text { troupeaux }\end{array}$ & $\begin{array}{l}\text { Nombre de bâtiments } \\
\text { construits }\end{array}$ & Quantitative \\
\hline & Investissements totaux & Réalisation d'investissements & Qualitative \\
\hline
\end{tabular}


Nous avons organisé les données dans une matrice $Z$ où les exploitations ( 50 individus au total) ont été caractérisées par les valeurs de chacun des 20 indicateurs retenus (20 variables quantitatives ou qualitatives selon leur nature) à chacune des six dates considérées (1955, 1965, 1975, 1985, 1995, 2005). Nous avons décomposé la variance de cette matrice selon la méthode statistique de Hill et Smith (1976) qui permet de considérer conjointement des données quantitatives et qualitatives dans un tableau. Cette méthode nous a permis d'analyser la variance de la matrice $\mathrm{Z}$ selon trois axes : les dates, les exploitations et leurs interactions. Parmi les six tableaux obtenus lors de cette opération, un tableau rend compte des valeurs des indicateurs pour chaque exploitation une fois l'effet moyen de la date enlevé, ce qui permet d'analyser les particularités de leur profil individuel d'évolution par rapport à la tendance moyenne de la population. Cette étape permet ainsi d'identifier les principaux changements communs aux exploitations sur la zone. La typologie de trajectoires a été construite à partir de ce dernier tableau selon une méthode d'analyse en deux étapes fréquemment utilisée pour l'élaboration de typologies (GarciaMartinez et al., 2009) : (i) le tableau a été soumis à une analyse en composantes principales (ACP), qui a permis de retenir quatre facteurs selon le critère de Kaiser (valeurs propres $>1$ ) et (ii) les coordonnées des exploitations sur les quatre premiers facteurs de l'ACP ont été utilisées pour effectuer une Classification Ascendante Hiérarchique $(\mathrm{CAH})$ selon la distance euclidienne et le critère d'agrégation de Ward. La classification a ensuite été stabilisée en utilisant la méthode des K-means avec les centroïdes des classes obtenues par la CAH fixés comme barycentres. Le calcul de la moyenne et de l'écart-type pour chaque variable quantitative a permis de décrire les différents types de trajectoires obtenus. Les analyses statistiques ont été réalisées avec le logiciel R 2.10.1® (R Development Core Team, 2011).

À partir des références bibliographiques (Veysset et al. 2005 ; Wilkins, 2008 ; Belland, 2011 ; Choisis et al., 2012) et des discussions avec les agriculteurs et le groupe local de partenaires, nous avons ensuite caractérisé les facteurs de l'environnement des exploitations à l'origine des changements observés. Cette analyse a été confrontée au regard des membres du groupe local de recherche en partenariat lors d'une réunion collective de discussion de la typologie de trajectoires. Douze entretiens individuels complémentaires conduits auprès des agriculteurs enquêtés ont donné lieu à une présentation et une discussion de notre typologie. Les agriculteurs nous ont alors permis de préciser notre analyse des différents types de trajectoires considérés. Pour évaluer les types de trajectoires ayant permis le maintien effectif de la polyculture-élevage, nous avons croisé notre typologie avec les types de systèmes de production présents en 2012. 


\section{Des trajectoires passées d'exploitations contrastées}

\section{Cinq types de " chemins pour durer » localement}

Les quatre premiers axes de l'ACP ont permis d'expliquer 63,1\% de la variabilité entre trajectoires. L'axe 1, qui explique $21,1 \%$ de la variabilité, correspond à un gradient d'intensification du système qui va des pratiques " traditionnelles " ${ }^{7}$ jusqu'à des systèmes de culture intensifiés. L'axe 2, qui explique $16,5 \%$ de la variabilité, correspond à un gradient qualifiant le choix d'orientation de l'élevage bovin vers la production laitière ou la production allaitante. L'axe 3, qui explique $15,1 \%$ de la variabilité, correspond à un gradient de système familial allant d'une organisation de type "famille-souche " à une organisation de type famille nucléaire ${ }^{8}$. L'axe 4, qui explique $10,4 \%$ de la variabilité, correspond à un gradient d'utilisation du sol majoritairement en prairies versus majoritairement en cultures de vente.

À partir de ces quatre facteurs principaux de différenciation des exploitations (système de pratiques, orientation bovine, système familial et utilisation du sol), la CAH a permis d'identifier cinq types de trajectoires, i.e. cinq types contrastés de " chemins pour durer" dans un même contexte (Fig. 2). Nous décrirons succinctement ces cinq trajectoires que nous qualifierons par un aphorisme qui peut certes paraître réducteur vis-à-vis de la complexité des évolutions réelles mais permet d'en résumer la stratégie.

7. Les pratiques dites «traditionnelles " correspondent ici à des systèmes de cultures cohérents avec les conditions de milieu peu favorables que rencontrent les agriculteurs des coteaux (faible réserve hydrique des sols, sécheresse estivale, forte pente dans certaines parcelles non mécanisables) : la rotation principale comprend des céréales à paille et des prairies, le maïs étant trop demandeur en eau ; les intrants (azote et produits phytosanitaires) sont apportés en quantité limitée, l'objectif n'étant pas de maximiser le rendement mais plutôt de produire de la manière la plus autonome possible.

8. Les systèmes familiaux peuvent s'organiser de différentes manières. Selon Emmanuel Todd, une organisation familiale nucléaire correspondrait à une organisation dans laquelle chaque ménage est autonome (père, mère et enfants). Dans une organisation familiale de type souche, l'un des fils demeure avec sa femme et ses enfants dans le foyer paternel en attendant la succession ; les ménages ne sont pas indépendants les uns des autres ( $C f$. Emmanuel Todd, L'Origine des systèmes familiaux. Tome I. L’Eurasie, Gallimard essais, 2011). 


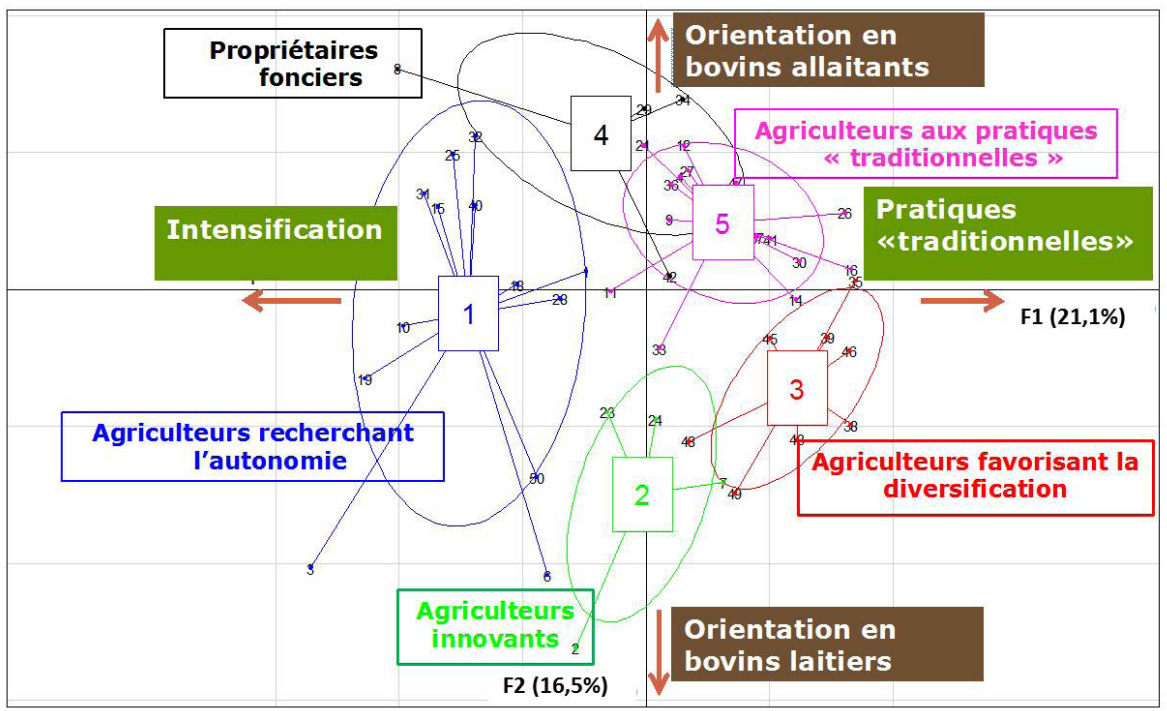

Fig. 2. Projection de la classification des trajectoires sur les deux premiers axes de l'ACP (adapté de Ryschawy et al., 2013).

Le premier chemin a consisté à rechercher la maximisation de l'autonomie par une coordination forte entre cultures et élevage au sein de l'exploitation. Ainsi, dans le type 1, pour durer " on n'est jamais mieux servi que par soi-même ».

Le deuxième chemin s'est basé sur la recherche permanente de l'innovation technologique. Ces agriculteurs ont toujours cherché à moderniser leur système et ont conduit des innovations de rupture (passage au bovin laitier en 1970, techniques culturales simplifiées dans les années 2000...) et ceci souvent dans une logique d'intensification de leur système de production. Il a conduit au développement d'exploitations laitières spécialisées. Dans le type 2, on considère que pour durer " qui ne tente rien, n'a rien".

Le troisième chemin s'est fondé sur une diversification des ateliers de production agricole pour bénéficier d'économies de gamme et se sécuriser par rapport aux fluctuations du marché des intrants et des produits agricoles. La vente directe ou l'accueil à la ferme ont fait partie des options de diversification mais aucune activité non liée à l'agriculture n'a été recensée. Dans le type 3, on pense que pour durer «il ne faut pas mettre tous ses œufs dans le même panier ".

Le quatrième chemin a été celui de la sécurisation de l'exploitation par l'acquisition de capital via l'agrandissement. Les agriculteurs du type 4 ont toujours extensifié leur système en innovant assez peu mais en favorisant des économies d'échelle. Dans le type 4, pour durer " abondance de biens ne nuit point ".

Enfin le cinquième et dernier chemin a reposé sur des adaptations progressives de pratiques " traditionnelles " en lien avec la main-d'œuvre familiale disponible. 
On estime ici que "l'union fait la force ", au sens que l'on est plus forts à plusieurs que tout seul. Dans ce type, la disponibilité en main-d'œuvre familiale a fortement conditionné les évolutions passées. Dans le sous-type 5-1, la main-d'œuvre disponible semble assurer le maintien des exploitations de la plupart des exploitations du type 5. Cette stratégie du type 5, très axée sur la mobilisation de la main-d'œuvre familiale, peut être largement limitante dans certains cas : si l'union fait la force, la désunion peut conduire à une dégradation de la résilience de l'exploitation. Cette stratégie peut ainsi parfois être " perdante " dans la mesure où le manque de maind'œuvre familiale conduit progressivement à la cessation d'activité d'une partie de ces exploitations (sous-type 5-2).

Le décompte du nombre d'exploitations encore en polyculture-élevage en 2012, au sein de chaque type de trajectoire, nous a permis de considérer que quatre chemins étaient compatibles avec le maintien de la polyculture-élevage. Seul un chemin exclut la polyculture-élevage : celui qui a conduit à la spécialisation et à l'intensification en production laitière. Toutefois, si la polyculture-élevage est présente dans les quatre autres trajectoires, elle devient partout minoritaire. Elle ne représente que quatre exploitations sur $13(31 \%)$ dans le type 1 ; trois exploitations sur huit (38\%), dans le type 3 ; deux exploitations sur six (33\%), dans le type 4 et quatre exploitations sur dix ( $40 \%$ ) dans le type $5-1$. Pour ces deux derniers types, le maintien de la polyculture-élevage est largement conditionné par la disponibilité en main- d'œuvre familiale.

\section{Des facteurs de contexte qui ont influé sur le maintien de la polyculture-élevage}

La figure 3 met en évidence les évolutions générales des exploitations sur le cas d'étude. Nous identifions trois principaux facteurs de changement qui ont favorisé le processus de spécialisation : (i) les incitations à la production de la PAC, (ii) la baisse tendancielle du prix des produits agricoles en lien avec la mondialisation des marchés et (iii) une main-d'œuvre agricole en diminution continue (Tab. 3). Certaines orientations politiques comme le développement des aides du second pilier de la PAC, pour favoriser le maintien de surfaces en prairies, ont limité l'abandon de l'élevage bovin par les agriculteurs locaux. Ces mesures ont dès lors favorisé indirectement la polyculture-élevage, en contrariant le processus de spécialisation vers les grandes cultures.

En plus des éléments de contexte, la diversité des trajectoires observées est aussi en partie liée à des différences de structures entre les exploitations en 1950. Certaines exploitations avaient des parcelles avec un plus fort potentiel à la modernisation, i.e. des parcelles drainables en fond de vallées, moins de parcelles en pente et non mécanisables, un parcellaire plus groupé. D’autres avaient, au contraire, une majorité de terres en pente et non mécanisables. Les exploitations du type 5 qui ont peu innové avaient en l'occurrence une majorité de terres en coteaux. 
Tab. 3. Facteurs de contexte impliqués dans le maintien de la polyculture-élevage (Ryschawy et al., 2013).

\begin{tabular}{|c|c|c|c|c|c|c|}
\hline Groupes de facteurs & \multicolumn{4}{|c|}{ Économie et politiques } & $\begin{array}{c}\text { Structure } \\
\text { d'exploitation }\end{array}$ & $\begin{array}{l}\text { Localisation } \\
\text { régionale }\end{array}$ \\
\hline \multirow[t]{2}{*}{$\begin{array}{c}\text { Facteurs de } \\
\text { changement impliqués }\end{array}$} & \multicolumn{2}{|c|}{$\begin{array}{l}\text { Mondialisation des } \\
\text { marchés agricoles }\end{array}$} & \multicolumn{2}{|c|}{$\begin{array}{c}\text { Orientations de la } \\
\text { PAC }\end{array}$} & $\begin{array}{c}\text { Diminution } \\
\text { de la main- } \\
\text { d'cuvre agricole }\end{array}$ & $\begin{array}{l}\text { Tradition de } \\
\text { polyculture- } \\
\text { élevage }\end{array}$ \\
\hline & $\begin{array}{c}\text { Prix } \\
\text { bas des } \\
\text { produits }\end{array}$ & $\begin{array}{l}\text { Fluctuations } \\
\text { des prix }\end{array}$ & $\begin{array}{l}\text { Aides } \\
\text { couplées }\end{array}$ & $\begin{array}{c}\text { Second } \\
\text { pilier }\end{array}$ & & \\
\hline $\begin{array}{c}\text { Impacts } \\
\text { sur le maintien de la } \\
\text { polyculture-élevage }\end{array}$ & - & + & - & + & - & + \\
\hline
\end{tabular}

-1950 - 1970 : «Systèmes à maison "

-Veaux sous la mère (race Gasconne), porcs et volailles avec auto-consommation de céréales et pâtures

$\rightarrow$ Exploitations diversifiées avec des systèmes de production comparables

-1970-1990 : Spécialisation des exploitations

-Innovations techniques et technologiques (races spécialisées, techniques

d'ensilage, ....),

-Nouvelles orientations liées au marché du maigre en Europe du Sud,

- Aménagement foncier des fonds de vallées (drainage, accès à l'irrigation, ...)

$\rightarrow$ Début de la modernisation et de l'intensification des pratiques agricoles
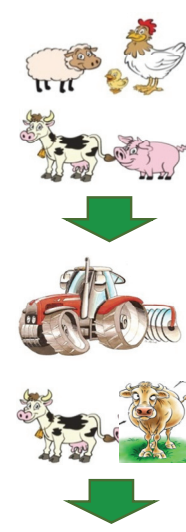

-1970-1990 : Agrandissement des exploitations

-Manque de main-d'œurre (problème de transmission/prix élevés du foncier)

-Développement de pratiques alternatives (Techniques Culturales Simplifiées, ...)

$\rightarrow$ Simplification des pratiques et cessations d'activité

Fig. 3. Synthèse des évolutions générales des exploitations sur la zone étudiée (adapté de Ryschawy, 2012). 


\section{Un éclairage nouveau des " chemins pour durer " en polyculture-élevage}

\section{Une diversité locale de trajectoires passées}

Notre analyse a permis de comprendre les facteurs de maintien et de transformation des systèmes de production dans un même contexte local et notamment des systèmes de polyculture-élevage. Malgré le maintien de ces systèmes dans la zone d'étude, les deux facteurs majeurs qui ont marginalisé les exploitations de polyculture-élevage en Europe (Veysset et al., 2005; Wilkins,2008) ont aussi joué un rôle localement. Le développement de l'agriculture européenne soutenu par les aides couplées de la PAC ainsi que la pression des marchés mondiaux ont incité à la spécialisation et à l'agrandissement des exploitations agricoles; processus favorables à l'intensification et aux économies d'échelles. Les primes à la production de céréales, relayées par les primes à la surface SCOP (surface céréales oléo-protéagineux) ainsi que les primes au drainage et à l'irrigation ont incité en particulier à la spécialisation en grandes cultures et à l'arrêt de l'élevage. Les systèmes de polyculture-élevage basés sur les économies de gamme (Vermersch, 2007) ${ }^{9}$ et non sur les économies d'échelle se sont trouvés défavorisés par ces orientations.

La polyculture-élevage a toutefois été maintenue par $49 \%$ des agriculteurs locaux. Les particularités culturelles des sociétés locales dites "à maison ", basées sur une polyculture-élevage d'autosubsistance traditionnelle et familiale et la transmission de la maison à un seul héritier (Sourdril et al., 2006) nous semblent, paradoxalement, avoir été source de maintien mais aussi de disparition de la polyculture-élevage. Ainsi l'attachement fort des agriculteurs au système de polyculture-élevage qui nous a été témoigné au cours des entretiens et des réunions a sans doute contribué à son maintien. Et ce d'autant que la diversité du type de terres présentes dans les maisons était un fondement de leur autonomie. Par contre, la faible démographie agricole générée par le système de transmission à un héritier unique a probablement accentué le problème de disponibilité de la main-d'œuvre, en particulier au cours de la période récente. L'emploi d'une main-d'œuvre essentiellement familiale et le faible recours au salariat, alors que la conduite conjointe d'ateliers diversifiés nécessite une main-d'œuvre importante, a limité le maintien de la polyculture-élevage. Cette réduction tendancielle de main-d'œuvre agricole a plus largement poussé à la simplification des pratiques, couplée à l'éventuelle recherche d'un revenu complémentaire à l'extérieur de l'exploitation. La double activité, qu'elle s'exerce dans ou hors secteur agricole, est défavorable au maintien de la polyculture-élevage en réduisant, parfois fortement, le temps de travail sur l'exploitation. Les trois agriculteurs double-actif de la zone ont en l'occurrence abandonné la polyculture-élevage pour se spécialiser en grandes cultures.

9. Chapitre 2 - Autonomie, hétéronomie, les fins et les moyens - Production autonome, hétéronome. 


\section{Des stratégies adaptatives locales en partie comparables à celles d'autres régions}

Lémery et al. (2005) ont mené une étude des comportements adaptatifs des agriculteurs en Bourgogne qui a mis en évidence des stratégies adaptatives dont certaines peuvent être rapprochées de celles des agriculteurs de notre zone d'étude. Sous une tendance " agir sur", ils ont identifié deux chemins : "efficience technique via l'optimisation et l'investissement dans le collectif " et "s'agrandir ", qui correspondent respectivement à nos types 2 et 4 . Sous une seconde tendance "faire avec ", ils en ont identifié deux autres : "rester autonome » et " diversifier avec d'autres activités ", qui correspondent respectivement à nos types 1 et $5-1$. Seul le type 3 , qui consiste à diversifier les productions de l'exploitation n'a pas été identifié car il est plus spécifique aux régions de polyculture-élevage. Garcia-Martinez et al. (2009) ont aussi trouvé, sur la période 1990-2004, des évolutions comparables en zone défavorisée de montagne (Pyrénées espagnoles). Une tendance d'évolution qualifiée " d'équilibre structurel " est observable sur les types 1 et 3 . Cette tendance correspond à une relative stabilité de la structure des exploitations sur la période. La tendance "fort agrandissement " qui s'exprime dans nos types 2 et 4 , correspond à de fortes augmentations de SAU et de taille du troupeau depuis 1990. Le type 5 n'a pas d'équivalence dans cette étude.

Rueff et al. (2012) ont aussi analysé des trajectoires d'exploitation en zone défavorisée de montagne (Pyrénées françaises). Ils ont identifié un type de trajectoire fondé sur une stratégie de gestion « patrimoniale » de biens agricoles familiaux ayant une forte proximité avec notre type 3 : un système à maison basé sur le maintien traditionnel d'activités agricoles diversifiées. Le type de trajectoire découlant d'une stratégie de " retrait " correspond à notre sous-type 5-2. En revanche, sur notre cas d'étude, le type de trajectoires correspondant à une stratégie de " niche ", fondée sur la mise en place de valorisations de type touristique, n'a pas été rencontré du fait du peu d'opportunités touristiques de la zone. Cette comparaison succincte avec d'autres travaux menés sur les trajectoires d'évolution des exploitations montre à la fois une certaine généricité des stratégies adaptatives des exploitations en France, et probablement en Europe, mais aussi des spécificités liées aux régions concernées.

\section{Limites et principaux intérêts de l'étude des trajectoires de changements passés}

Comme Rueff et al. (2012), nous avons choisi d'initier l'étude des trajectoires de changement des exploitations à partir de 1950 pour des raisons scientifiques et méthodologiques. La modernisation des exploitations européennes a débuté en 1950 : les trajectoires ont donc connu des inflexions majeures à partir de cette période (Antrop, 2005). Mais cinquante ans apparaissent aussi comme une limite dans la mémoire des familles agricoles lors de la collecte de données rétrospectives (Demazière et Dubar, 1997 ; Mottet et al., 2006 ; Cialdella et al., 2009) ; les données étant souvent limitées par la précision de l'information obtenue pendant les 
entretiens. À titre d'exemple, les agriculteurs vont facilement se souvenir de la présence d'une culture sur les décennies passées, mais plus difficilement de la superficie cultivée. Comme toute personne, les agriculteurs " reconstruisent le passé ", même s'ils essaient d'être objectifs (Lamine et Bellon, 2008). L'expérience modifie l'interprétation des faits (Cialdella et al., 2009).

Nous avons ainsi accordé de l'importance à l'interprétation des données par les agriculteurs bien qu'ils fassent néanmoins preuve d'une inévitable subjectivité. Nous considérons que les méthodes d'analyses statistiques informatisées nous ont permis de limiter cette subjectivité dans notre étude (Mulaik, 1993). L'analyse statistique a été choisie sur la base de considérations conceptuelles et empiriques.

Comme l'ont souligné Moulin et al., 2008, l'étude de trajectoires passées des exploitations est un matériau précieux pour la compréhension des stratégies adaptatives des agriculteurs mises en place sur le temps long (hypothèse de continuité). La dimension historique est fondamentale pour appréhender l'évolution des exploitations et doit aussi servir de base pour l'avenir (Capillon et Manichon, 1979). Van der Ploeg et al. (2009) ont d'ailleurs montré que certaines petites exploitations pouvaient durer, en opposition à l'idée que toutes les exploitations qui ne remplissaient pas les exigences du modèle de modernisation allaient disparaître. Les " chemins pour durer » suivis par les exploitations leur auraient en effet permis de développer des capacités adaptatives susceptibles de les aider à se maintenir à l'avenir, en contexte incertain (Meinke et al., 2009 ; Darnhofer et al., 2010). Scoones (2009) souligne le besoin urgent d'élaborer de nouveaux outils pour enrichir ce type d'approches, nécessaires pour aborder les questions complexes du développement rural.

Enfin, les ateliers de travail avec les partenaires locaux et des échanges individuels avec certains agriculteurs nous ont permis d'approfondir nos interprétations des types de trajectoires obtenus et de confirmer les tendances et éléments de contexte que nous avions identifiés. Cette démarche illustre l'intérêt d'une collaboration avec les acteurs locaux (Gibon et al., 1999b et 2010).

\section{Conclusion}

Les incitations à la production de la PAC, les faibles cours des produits agricoles alliés à une diminution de la main-d'œuvre agricole disponible ont concouru à marginaliser les systèmes de polyculture-élevage en Europe, tout en favorisant le processus de spécialisation. Néanmoins, dans la région étudiée, les choix des agriculteurs en partie fondés sur des valeurs culturelles ancrées localement ont contribué à maintenir des exploitations de polyculture-élevage.

Notre typologie met en lumière cinq types de trajectoires des exploitations sur le site d'étude. Parmi ceux-ci, quatre " chemins pour durer » ont permis le maintien d'exploitations de polyculture-élevage. Nous faisons l'hypothèse que les stratégies d'adaptation futures des agriculteurs s'inscriront dans la continuité des chemins 
passés favorables au maintien des systèmes de polyculture-élevage (Moulin et al., 2008).

Compte tenu de l'incertitude du contexte politique avec la réforme en cours de la PAC et l'instabilité des marchés agricoles, deux types de stratégies adaptatives nous semblent particulièrement intéressantes à considérer dans une perspective de durabilité des exploitations, en général, et de polyculture-élevage, en particulier : les chemins " maximisation de l'autonomie " et "diversification ". Autonomie et diversification sont aussi deux stratégies majeures débattues au sein des différentes arènes abordant le futur de l'agriculture dans leur capacité à concilier durabilité économique et environnementale et viabilité des exploitations.

L'autonomie est ainsi au cœur des préoccupations des agriculteurs engagés dans des démarches plus économes et d'agriculture durable. Une forte coordination entre cultures et élevage est un des moyens pour sécuriser l'alimentation des animaux et entretenir la fertilité organique des sols vis-à-vis des fluctuations du marché des intrants mais aussi pour bénéficier d'économies de gamme. Suite à cette étude, nous avons engagé, avec le groupe local de recherche en partenariat, une réflexion prospective sur la co-construction de scénarios potentiellement favorables au maintien de la polyculture-élevage dans les coteaux de Gascogne (Ryschawy et al., 2014).

\section{Remerciements}

Cette étude a été réalisée dans le cadre d'une thèse, effectuée au sein de l'UMR 1201 Dynafor, Inra-INPT/Ensat et IE Purpan. Les auteurs tiennent à remercier pour son soutien l'Agence nationale de la recherche, dans le cadre des projets BiodivagriM (ANR-07-BDIV, 2008-2012) et Mouve (ANR-2010-STRA-005 MOUVE, 20112016). Les auteurs remercient aussi la Région Midi-Pyrénées et l'INRA, dans le cadre du projet « Changement d'utilisation agricole et forestière des terres et dynamique des paysages agriforestiers de Midi-Pyrénées » (CHAPAY) du programme de recherche "Pour et Sur le Développement Régional en Midi- Pyrénées » (PSDR, 2008-2011).

\section{Bibliographie}

Agreste, 2010. Tableau standard RICA 2002-2008, Rapports Publics-RICA Réseau d'Information Comptable Agricole.

Antrop, M., 2005. Why landscapes of the past are important for the future ? Landscape and Urban Planning, 70, 1, 21-34.

Belland, C., 2011. Étude de l'influence des marchés et des politiques publiques agricoles sur la dynamique des systèmes de polyculture-élevage dans les coteaux de Gascogne, Master Pro ERG Agrocampus-Ouest. 
Capillon, A., Manichon, H., 1979. Une typologie des trajectoires d'évolution des exploitations agricoles (principes, application au développement agricole régional), Comptes-rendus des séances de l'Académie d'agriculture de France, 1168-1178.

Chatellier, V., Gaigné, C., 2012. Les logiques économiques de la spécialisation productive du territoire agricole français, Innovations Agronomiques, 22, 185-203.

Choisis, J.-P., Sourdril, A., Deconchat, M., Balent, G., Gibon A., 2010. Understanding regional dynamics of mixed crop-livestock agricultural systems to support rural development in South-western France uplands, Cahiers Agricultures, 19, 2, 97-103.

Choisis, J.-P., Thévenet, C., Gibon, A., 2012. Analyzing farming systems diversity : a case study in south-western France, Span J Agric Res, 10, 3, 605-618.

Cialdella, N., Dobremez, L., Madelrieux, S., 2009. Livestock farming systems in urban mountain regions. Differentiated paths to remain in time, Outlook on Agriculture, 38, 2, 127-135.

Darnhofer, I., Bellon, S., Dedieu, B., Milestad, R., 2010. Adaptiveness to enhance the sustainability of farming systems, A review, Agronomy for Sustainable Development, 30, 3, 545-555.

Dedieu, B., 2009. Qualification of the adaptative capacities of livestock farming systems, Revista Brasileira de Zootecnia, 38, 397-404.

Demazière, D., Dubar C., 1997. Analyser les entretiens biographiques. L'exemple de récits d'insertion, Paris, Nathan.

Dobremez, L., Bousset, J.-P., 1996. Rendre compte de la diversité des exploitations agricoles. Une démarche d'analyse par exploration conjointe de sources statistiques, comptables et technico-économiques, Gestion du territoire $\mathrm{n}^{\circ} 17$, éditions du Cemagref.

Dolédec, S., Chessel, D., 1987. Rythmes saisonniers et composantes stationnelles en milieu aquatique. I- Description d'un plan d'observations complet par projection de variables, Acta Oecologica, Oecologia Generalis, 8, 3, 403-426.

EC (European Commission), 1999. Specialised holdings and more intensive practices. Available at : ec.europa.eu/agriculture/envir/report/en/expl_en/report.htm Accessed January 2014.

FAO, 1995. Digital Soil Map of the World and Derived Soil Properties. Land and Water Digital Media Series, 7, Food and Agriculture Organization of the United Nations, Rome.

Garcia-Martinez, A., Olaizola, A., Bernués, A., 2009. Trajectories of evolution and drivers of change in European mountain cattle farming systems, Animal, 3, 1, 152-165.

Gibon, A., Balent, G., Olaizola, A., Di Pietro, F., 1999a. Approche des variations communales des dynamiques rurales au moyen d'une typologie : cas du versant nord des Pyrénées Centrales, Options Méditerranéennes, 27(B),15-34.

Gibon, A., Sheeren, D., Monteil, C., Ladet, S., Balent, G., 2010. Modelling and simulating change in reforesting mountain landscapes using a social-ecological framework, Landscape Ecology, 25, 2, 267-285. 
Gibon, A., Sibbald, A.R., Flamant, J.C., Lhoste, P., Revilla, R., Rubino, R., Sorensen, J.T., 1999b. Livestock farming systems research in Europe and its potential contribution for managing towards sustainability in livestock farming, Livestock Production Science, 61, 2-3, 121-137.

Hill, M.O., Smith, A.J.E., 1976. Principal component analysis of taxonomic data with multi-state discrete characters, Taxon, 25, 249-255.

Lamine, C., Bellon, S., 2008. Conversion to organic farming : a multidimensional research object at the crossroads of agricultural and social sciences, A review, Agronomy for Sustainable Development, 29, 1, 97-112.

Lémery, B., Ingrand, S., Dedieu, B., 2005. Agir en situation d'incertitude : le cas des éleveurs bovins allaitants, Économie Rurale, 288, 57-69.

Mazoyer, M., Roudart, L., 1997. Histoire des agricultures du monde. Du Néolithique à la crise contemporaine (nouvelle édition revue et augmentée, Paris, Seuil, collection Pont Histoire, 2002).

Meinke, H., Howden, S.M., Struik, P.C., Nelson, R., Rodriguez, D., Chapman, S.C., 2009. Adaptation science for agricultural and natural resource management - Urgency and theoretical basis, Curr. Opin. Environ. Sustain., 1, 1, 69-76.

Meynard, J.-M., 2012. Conclusion In CIAG, Associer productions animales et végétales pour des territoires agricoles performants, 24 octobre 2012, Poitiers, France.

Milestad, R., Darnhofer, I., 2003. Building farm resilience : the prospects and challenges of organic farming, Journal of Sustainable Agriculture, 22, 3, 81-97.

Mottet, A., Ladet, S., Coqué, N., Gibon, A., 2006. Agricultural land-use change and its drivers in mountain landscapes: A case study in the Pyrenees, Agriculture, Ecosystems \& Environment, 114, 2-4, 296-310.

Moulin, C.-H., Ingrand, S., Lasseur, J., Madelrieux, S., Napoléone, M., Pluvinage, J., Thénard, V., 2008. Comprendre et analyser les changements d'organisation et de conduite de l'élevage dans un ensemble d'exploitations : propositions méthodologiques, in Dedieu, B., Chia, E., Leclerc, B., Moulin, C.-H. (Eds), L'élevage en mouvement: flexibilité et adaptation des exploitations d'herbivores, Paris, Éditions Quae, 181-196.

Mulaik S.A. 1993. Objectivity and Multivariate Statistics, Multivariate Behavioral Research, 28, 2, 171-203.

R Development Core Team, 2011. R : A language and environment for statistical computing. R Foundation for Statistical Computing, Vienna, Austria. URL http://www.R-project.org.

Rueff, C., Choisis, J.P., Balent, G., Gibon, A., 2012. A preliminary assessment of the local diversity of family farms change trajectories since 1950 in a Pyrenees mountains area, Journal of Sustainable Agriculture, 36, 5, 564-590.

Russelle, M.P., Entz, M.H., Franzluebbers, A.J., 2007. Reconsidering integrated crop-livestock systems in North America, Agronomy Journal, 99, 325-334.

Ryschawy, J., 2012. Éclairer les conditions de maintien d'exploitations de polycultureélevage durables en zone défavorisée simple européenne. Une étude de cas dans les coteaux de Gascogne, Thèse de doctorat. INP-Université de Toulouse. 
Ryschawy, J., Choisis, N., Choisis, J.-P., Joannon, A., Gibon, A., 2012. Mixed croplivestock systems : an economic and environmental-friendly way of farming ? Animal, 6, 10, 1722-1730.

Ryschawy, J., Choisis, N., Choisis, J.-P., Gibon, A., 2013. Paths to last in mixed crop-livestock farming : lessons from an assessment of farm trajectories of change, Animal, 7, 4, 673-681.

Ryschawy, J., Joannon, A., Choisis, J.P., Gibon, A., Le Gal, P.Y., 2014. Participative assessment of innovative technical scenarios for enhancing sustainability of French mixed crop-livestock farms, Agricultural systems, 129, 1-8.

Scoones, I., 2009. Livelihoods perspectives and rural development, Journal of Peasant Studies, 36, 1, 171-196.

Sourdril, A., Du Bus de Warnhaffe, G., Deconchat, M., Garine, E., Balent, G., 2006. From farm forestry to farm and forestry in South-Western France as a result of changes in a 'house-centred' social structure, Small-scale forest, economics, management, and policy, 5, 1, 127-144.

Van Der Ploeg, J.D., Laurent, C., Blondeau, F., Bonnafous, P., 2009. Farm diversity, classification schemes and multifunctionality, J. Environ. Manage., 90, S2, S124-S131.

Vermersch, D., 2007. L'éthique en friche, Paris, Éditions Quae.

Veysset, P., Bebin, D., Lherm, M., 2005. Adaptation to Agenda 2000 (CAP reform) and optimisation of the farming system of French suckler cattle farms in the Charolais area : a model-based study, Agricultural Systems, 83, 2,179-202.

Wilkins, R.J., 2008. Eco-efficient approaches to land management : a case for increased integration of crop and animal production systems, Philosophical Transactions of the Royal Society B-Biological Sciences, 363, 1491, 517-52. 